\title{
Indicadores Demográficos y Estimación de la Población de canes con dueño en el distrito de San Martin de Porres, Lima-Perú
}

\author{
Demographic Indicators and Estimated Population of owned dogs in the district of San Martin de Porres, \\ Lima-Peru
}

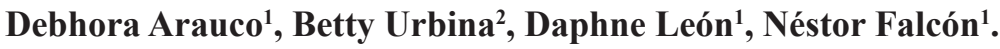

\section{RESUMEN}

Objetivo: Determinar los indicadores demográficos y estimar la población de canes con dueño en el distrito de San Martin de Porres. Metodología: Se desarrolló encuestas, que se entregaron a los hogares a través de los estudiantes de nivel primario o fueron resueltas en el aula por estudiantes de nivel secundario de Instituciones Educativas cuyas autoridades respaldaron la investigación. El instrumento utilizado fue aprobado por las autoridades de la Municipalidad, las mismas que apoyaron la coordinación y distribución correspondiente. Para la estimación de la población de canes se utilizó los resultados del Censo de población del INEI del año 2007 y la proyección de esta población al 2013. Resultados y Conclusiones: Se encontró que el porcentaje de hogares con canes fue de $58,2 \%$ y que la media de canes por vivienda fue de 1,6. La relación persona:can fue 7:1. Con datos del censo del INEI del 2007, la proyección estimada total de canes que fue de 82 794. La estimación de perros ajustado al tipo de vivienda y la relación persona:can fue de 83 845, difiriendo en 1051 (1.01\%). Considerando la proyección del crecimiento poblacional para el año 2013 y manteniendo la relación persona:can, se estimó un total de 96164 canes lo que representaría una tasa de crecimiento de $16,1 \%$ para el periodo $2007-2013$. Se espera que los resultados del estudio ofrezcan información que favorezca la labor del sector salud y autoridades municipales en el desarrollo y planificación de las campañas de vacunación antirrábica y los programas relacionados con la tenencia responsable de los animales de compañía.

Palabras claves: estimación población de canes, San Martin de Porres, tenencia responsable de animales de compañía

\begin{abstract}
Aim: The aim of this study was to determine demographic indicators and to estimate the population of owned dogs in the district of San Martin de Porres using surveys. Methods: This was carried out with the help of school students under authorities approval and supervision. To calculate the dog's population we used the INEI's Population Census from 2007 and their projection to the year 2013. Results and Conclusions: Results showed that the percentage of households with dogs was $58,2 \%$, the average per house was $1,6 \mathrm{dogs}$, and the human:can ratio was $7: 1$. Using the INEI's census data from 2007, the total dog's population estimated was 82794 , however we estimated the dogs' population, adjusted to the type of housing and the human: can ratio, to be 83845 , which differed in $1051(1.01 \%)$. Taking in consideration the projected population growth by 2013, and keeping the human: can ratio, there will be a total of 96164 dogs which could represent a growth rate of $16,1 \%$ for 2007 to 2013 . The results of this study will provide valuable information to promote the work from the health and local authorities to plan rabies vaccination campaigns and to develop programs related to a responsible pet ownership in the community.
\end{abstract}

Keywords: dog population, San Martin de Porres, responsible pet ownership.

Facultad de Medicina Veterinaria y Zootecnia - Universidad Peruana Cayetano Heredia

Municipalidad de San Martin de Porras 


\section{INTRODUCCIÓN}

La convivencia de canes con el hombre ha demostrado indudables beneficios económicos y culturales; sin embargo, también se han encontrado desventajas para la salud, bienestar y la seguridad de las personas, sobre todo en zonas donde su tenencia y reproducción no es controlada (Güttler, 2005), constituyendo una preocupación para las autoridades sanitarias, al reconocer que la sobrepoblación de canes tiene un efecto directo en la salud de las personas, pudiendo transmitir más de 100 enfermedades zoonóticas (Ortega, 2001).

De todas las enfermedades zoonóticas que se puede contraer a través del contacto con el can, la de mayor importancia es la rabia (Acha y Szyfres, 2001).Esta enfermedad afecta principalmente a las poblaciones con acceso desigual a los servicios de salud. A lo largo de la historia la mayoría de casos de rabia humana transmitida por canes se ha producido en poblaciones vulnerables, caracterizadas por condiciones de vida y de aspecto laboral bajas, donde además se encuentra un alto porcentaje de canes que no han sido vacunados contra la rabia canina (REDIPRA, 2013).

La rabia sin embargo, a pesar de ser una enfermedad mortal, es prevenible mediante la vacunación. Actualmente, se vacunan todos los años alrededor de 44 millones de canes en Latinoamérica, situando a la vacunación canina como la estrategia de control más importante para detener la circulación del virus (REDIPRA, 2010).Ya que la rabia es una enfermedad fatal, es imprescindible el manejo de las poblaciones de canes para su control (WSPA, 2007).

La falta de conciencia del ser humano origina una tenencia irresponsable de mascotas, causando principalmente que la población de canes aumente de manera exponencial (Butcher, 2000). Los estudios demográficos y la estimación de la población de canes se presentan como una herramienta importante para la planificación de vacunación y tenencia responsable de estos animales. El primer estudio en este aspecto se realizó en Chile (Montes, 1966). Posteriormente se han publicado datos aislados sobre el tamaño de la población canina en algunos países y/o ciudades, mayormente obtenidas de encuestas específicas realizadas en localidades que desean conocer la dinámica poblacional de los canes con el objetivo de mantenerlos en mejores condiciones e implementar medidas de control (Álvarez y Domínguez, 2001).
La estimación de poblaciones de canes permite calcular los recursos necesarios para llevar a cabo programas y evaluar los resultados obtenidos (Martin, et al., 1997). Estos programas deben considerar la educación y legislación sobre la tenencia responsable, registro e identificación de los canes, control reproductivo, adopción, control del movimiento de los canes (normas sobre el uso de correa o la presencia de perros vagabundos), etc. (OIE, 2009).

El conocimiento de la cantidad aproximada de canes con dueño en cada distrito de Lima es importante, debido a que a partir de ella las autoridades del sector salud y municipales podrán mejorar la planificación y desarrollo de las campañas de vacunación de canes y los programas de tenencia responsable. Para ello existen una serie de metodologías las cuales requieren de una importante inversión en recursos económicos, humanos y logísticos, que en la actualidad no se han considerado dentro de los presupuestos necesarios en estas instituciones (salud y municipales).

En este contexto, considerando que las Instituciones Educativas (IE) son lugares donde se reúnen miembros de un gran número de hogares de la comunidad (escolares), y que estas pueden ser utilizadas para hacer llegar una encuesta a los jefes de familia o pueden ser desarrolladas por estudiantes. El objetivo del estudio fue estimar la población de canes con dueño en el distrito de San Martin de Porres (SMP) a través del desarrollo de encuestas en Instituciones Educativas Estatales (IEE) e Instituciones Educativas Particulares (IEP) del distrito.

\section{MATERIALES Y MÉTODOS}

\section{Lugar de Estudio}

El estudio se desarrolló en IEE y IEP del distrito de SMP. La elaboración de la base de datos y el análisis estadístico se realizó en la Facultad de Medicina Veterinaria y Zootecnia de la Universidad Peruana Cayetano Heredia (FAVEZ-UPCH)

\section{Diseño del estudio}

La investigación correspondió a un estudio observacional, descriptivo y transversal.

\section{Consideraciones éticas}

Las IEE e IEP participaron en el estudio previa aceptación de los directores. A fin de proteger identidad de personas e instituciones (por solicitud expresa de la mayoría de directores) las encuestas eran anónimas y el nombre de las IE participantes se mantuvo en reserva. 
La participación de los encuestados fue voluntaria.

\section{Población de estudio, tamaño de muestra y tipo de muestreo}

La población que se estudió fueron los hogares de escolares de IEE e IEP del distrito de SMP. Para determinar el número de IEE e IEP involucradas en el estudio se tomó en cuenta las zonas en las que políticamente se encuentra dividido el distrito. El estudio involucró al $25 \%$ de las zonas delimitadas. Estas áreas fueron enumeradas en forma correlativa y la selección de las mismas se realizó siguiendo las características de aleatoriedad basadas en un muestreo sistemático.

La selección de la zona de arranque se determinó en forma aleatoria entre los números del 1 al 4 . Obtenido el número del sector de arranque, se le sumó una constante $(n=4)$, hasta completar el tamaño de sectores especificados (25\% del total de sectores en los que se encontraba dividido el distrito).

Para determinar las IE que se incluyeron en el estudio, en cada uno de los sectores seleccionados, se requirió la siguiente información:

- Número de instituciones educativas estatales y privadas en cada sector

- Población aproximada de estudiantes en cada Institución Educativa

Dicha información sirvió para determinar las IEE e IEP con mayor población estudiantil (las más grandes) que participaron en el estudio. Bajo este criterio, el número de IEE e IEP que se consideraron en el estudio fueron 14 por cada tipo de IE.

\section{Variables en estudio}

Las variables que se consideraron en el estudio fueron:

- Distrito donde vive

- Tipo de vivienda (casa o departamento)

- Número de personas por hogar

- Número de niños en edad escolar (control de encuesta en hogar)

- Tenencia de canes (si o no)

- Registro de canes en la municipalidad(si o no)

- Número de canes en el hogar y características demográficas (sexo, edad, raza).

- Tenencia de animales castrados

- Vacunación antirrábica en el último año

- Desparasitación en el último año (número de veces)

- Método de control de la reproducción utilizado

- Nivel de confinamiento (permanente, temporal, sin confinamiento)

El instrumento fue validado por expertos y fue aceptado por el responsable del área de zoonosis de la Municipalidad del Distrito de SMP.

\section{Recolección de los datos}

Se coordinó con las diferentes IEE e IEP seleccionadas para el desarrollo del estudio, la participación en el proyecto y la fecha de envío o desarrollo de las encuestas. Las metodologías para la obtención de la información fueron dos, las cuales eran mutuamente excluyentes para cada nivel de educación de la IE participante: (1) las encuestas fueron enviadas a los hogares a través del cuaderno de control de los estudiantes, debiendo ser devueltas por la misma vía en un plazo no mayor de una semana en el caso de estudiantes de nivel primario, y (2) las encuestas fueron contestadas por los alumnos del nivel secundario. Se consideró una sola encuesta por familia.

La información acerca de la población de habitantes y el total de viviendas del distrito de SMP fue solicitada al Instituto Nacional de Estadística e Informática (INEI). Se requirieron los datos del Censo Nacional de Población y Vivienda del año 2007 y las proyecciones al año 2013 hechas por la misma institución el año 2009.

\section{Procesamiento de los datos}

La información recogida de la revisión de las encuestas fue transferida a una base de datos en el programa Microsoft Excel utilizando el lenguaje numérico. Después de culminada la base de datos inicial, se realizó una revisión completa de la información introducida obteniéndose la base definitiva para realizar el análisis de datos correspondiente.

\section{Análisis de los datos}

La base de datos en formato Excel se procesó con el software SPSS Statistics 17,0. Se calculó la proporción de viviendas del distrito que poseen canes, el promedio de canes por vivienda y la relación persona:can.

El cálculo de la población de canes estimada (PCE) se realizó con la información de la población humana del distrito y la relación persona:can a través de la siguiente fórmula:

PCE $=$ Nro. Hab. $/$ Relación persona:can

Dónde: 
Nro. Hab. $=$ Total de habitantes del distrito según el último censo nacional del $2007 \mathrm{y}$ las proyecciones al 2013.

Relación persona:can $=$ estimado del número de personas por cada can calculado en el estudio.

Las características demográficas y de manejo se resumieron en tablas de frecuencia.

\section{RESULTADOS}

Luego del proceso de depuración de la información, se obtuvieron 1947 encuestas válidas. La distribución de las mismas según tipo de vivienda, cantidad de personas que habitan la vivienda y tenencia de canes se presenta en el cuadro 1.

La media de personas por vivienda fue de 6,6 (desviación estándar $=3,72$, mediana $=5$, moda $=5$ ). El total de personas registradas en las 1947 encuestas válidas fue de 12765 . El número medio de canes por vivienda fue de 1,6 (desviación estándar $=1,2$, mediana $=1$, moda = 1). El 58,2 \% (1134) de las encuestas indicaron que tenían un can en la vivienda. El total de canes registrados fue de 1828. A partir de esta información se obtuvo una relación persona:can de 7:1 para SMP.

Entre las que vivían en departamentos solo el 35,6\% (98/275) tenían canes en contraste a quienes vivían en casas donde el 64,3\% (978/1521) tenían canes. No se consideró en este cálculo otro tipo de respuesta. El cuadro 2 muestra los indicadores de tenencia de canes distribuidos según el tipo de vivienda. En el cuadro 3 se muestra la estimación de canes para el distrito, utilizando la relación persona:can y la población de habitantes según el Censo de población del INEI 2007 distribuidos por tipo de vivienda.

Cuando se estimó la población de canes con el total de habitantes se obtuvo un estimado de 82794 canes para el distrito (año 2013), encontrándose una diferencia respecto a la estimación de la población de canes ajustada al tipo de vivienda (total estimado 83845 canes). La diferencia entre ambos cálculos fue de 1051 canes que correspondió a una variación porcentual de $1,01 \%$. Cuando se estimó la población de canes con la información de la proyección de personas que habitaban en el 2013 en SMP, se observó que la población humana estimada estaba en 673149 lo que habría correspondido a un total de canes de 96 164. Esto representaría una tasa de crecimiento de canes de 16,1\% entre el 2007 y el 2013.

La distribución según número y utilidad del can en la vivienda y el tiempo de confinamiento de los mismos se presenta en el cuadro 4 .

Sobre un total de 518 respuestas en la que indicaron la edad en la que falleció su ultimo can, se calculó que en SMP, la media de vida de los canes era de 5,2 años (desviación estándar $=4,31$, mediana $=4$, moda $=2$ ). Los valores extremos muestran animales que fallecen desde el mes de edad hasta los 21 años.

A partir de la descripción de las características de los canes que en la actualidad poseen los encuestados, se encontró información de la edad de 1676 canes. El promedio de edad de estos animales fue de 2,7 años

Cuadro 1. Distribución de las encuestas válidas distribuido por tipo de vivienda, cantidad de personas que habitan la vivienda y tenencia de canes. San Martín de Porres. $2013(\mathrm{n}=1947)$

\begin{tabular}{cccc}
\hline VARIABLE & ESTRATOS & NÚMERO & PORCENTAJE \\
\hline \multirow{2}{*}{ Tipo de vivienda } & Casa & 1521 & 78,1 \\
& Departamento & 275 & 14,1 \\
& Otros & 141 & 7,3 \\
No contestó & 10 & 0,5 \\
Número de personas por & Hasta 3 personas & 236 & 12,1 \\
vivienda & 4 a 5 personas & 759 & 39,0 \\
& 6 a 10 personas & 719 & 36,9 \\
11 a 15 personas & 173 & 8,9 \\
Más 15 personas & 60 & 3,1 \\
& & & \\
& No & 813 & 41,8 \\
\hline
\end{tabular}


Cuadro 2. Indicadores de tenencia de canes distribuidos según el tipo de vivienda. San Martín de Porres. 2013.

\begin{tabular}{|c|c|c|c|c|c|c|c|}
\hline \multirow{2}{*}{ Tipo de vivienda } & \multirow{2}{*}{$\begin{array}{c}\text { Total de } \\
\text { viviendas }\end{array}$} & \multirow{2}{*}{$\begin{array}{l}\text { Total de } \\
\text { personas }\end{array}$} & \multicolumn{2}{|c|}{ Viviendas con can(es) } & \multirow{2}{*}{$\begin{array}{c}\text { Total de } \\
\text { Canes }\end{array}$} & \multirow{2}{*}{$\begin{array}{c}\text { Promedio } \\
\text { de canes/ } \\
\text { vivienda }\end{array}$} & \multirow{2}{*}{$\begin{array}{c}\text { Relación } \\
\text { persona: } \\
\text { can }\end{array}$} \\
\hline & & & Nro. & $\%$ & & & \\
\hline Casa & 1521 & 10578 & 978 & 64,3 & 1613 & 1,65 & $6,6: 1$ \\
\hline Departamento & 275 & 1358 & 98 & 35,6 & 139 & 1,42 & $9,8: 1$ \\
\hline Otros & 151 & 829 & 58 & 38,4 & 76 & 1,31 & $10,9: 1$ \\
\hline Total & 1947 & 12765 & 1134 & 58,2 & 1828 & 1,61 & $7,0: 1$ \\
\hline
\end{tabular}

Cuadro 3. Población de canes estimada según censo de población del INEI - 2007. San Martín de Porres. 2013.

\begin{tabular}{cccc}
\hline Tipo de vivienda & Total personas Censo INEI & $\begin{array}{c}\text { Relación } \\
\text { Persona: can }\end{array}$ & Total de canes estimado \\
\hline Casa & 2007 & $6,6: 1$ & 76230 \\
Departamento & 503117 & $9,8: 1$ & 5972 \\
Otros & 58530 & $10,9: 1$ & 1643 \\
Total & 17914 &.-- & 83845 \\
\hline
\end{tabular}

Cuadro 4. Distribución de los tenedores de canes según número y utilidad del can en la vivienda y el tiempo de confinamiento. San Martín de Porres. 2013 ( $\mathrm{n}=1134)$

\begin{tabular}{lccc}
\hline Variable & Estratos & Número & Porcentaje \\
\hline & Uno & 741 & 65,3 \\
Número de canes & Tres & 256 & 22,6 \\
por vivienda & 4 a 5 & 61 & 5,4 \\
& 6 a 10 & 58 & 5,1 \\
& 11 a 15 & 17 & 1,5 \\
& Mascota & 1 & 0,1 \\
Utilidad del & Guardián & & \\
Can & Reproducción - Venta & 825 & 72,7 \\
& Mascota/Guardián & 131 & 11,5 \\
& Mascota/Reproducción-venta & 3 & 0,7 \\
& No contestó & 71 & 8,5 \\
Confinamiento de & & & 0,3 \\
los canes & Todo el día en casa & 254 & 6,3 \\
& Sale a momentos solos & 332 & 22,4 \\
& Sale con supervisión & 412 & 29,3 \\
& Sale todo el día & 67 & 36,3 \\
& No contestó & 69 & 5,9 \\
\hline
\end{tabular}


Cuadro 5. Características demográficas de los canes que en la actualidad poseen los encuestados. San Martín de Porres. 2013

\begin{tabular}{cccc}
\hline Variable & Estratos & Número & Porcentaje \\
\hline Sexo & Macho & 939 & 56,6 \\
$(\mathrm{n}=1659)$ & Hembra & 720 & 43,4 \\
& & & \\
Raza & Puro & 753 & 45,9 \\
$(\mathrm{n}=1640)$ & Cruzado & 887 & 54,1 \\
& & & \\
Tamaño & Pequeño & 598 & 38,3 \\
$(\mathrm{n}=1562)$ & Mediano & 659 & 42,2 \\
& Grande & 305 & 19,5 \\
Castrado o & & & \\
esterilizado & No & 1388 & 88,4 \\
$(\mathrm{n}=1570)$ & Si & 182 & 11,6 \\
\hline
\end{tabular}

(desviación estándar $=2,75$, mediana $=2$, moda $=1)$.En el cuadro 5 se observa las características demográficas de los canes en la actualidad, según lo registrado en las encuestas.

En 193 encuestas se encontró el registro de camadas en el último año. El 63,7 \% (123) mencionó que su can tuvo una camada, 26,9\% (52) mencionaron que tuvieron dos camadas y $9,3 \%$ (18) mencionó que su perra tuvo tres camadas en el último año. Se registró un total de 1058 crías nacidas, de las cuales sobrevivieron 801 lo que correspondió a una tasa de sobrevivencia del $75,7 \%$, y consecuentemente una tasa de mortalidad del $24,3 \%$.

\section{DISCUSIÓN}

Una de las principales deficiencias que tienen los programas de control de rabia y de tenencia responsable de animales de compañía (TRAC) es el desconocimiento de la población de canes. La realización de censos no ha sido considerada dentro de las tareas prioritarias para superar esta deficiencia por las autoridades de salud y gobiernos locales, debido al costo y los requerimientos logísticos que implican. Por ello, los estudios de estimación de poblaciones de estos animales resultan una alternativa económica que permitiría superar parte de este problema.

La estimación de la población de canes es de importancia para la planificación adecuada de las campañas antirrábicas (VANCAN), las cuales se encuentran inmersas dentro del programa de control, prevención y erradicación de esta zoonosis. Además esta información sirve de línea de base para el desarrollo de programas de TRAC que los gobiernos locales están obligados a realizar en el marco del cumplimiento de la Ley No 27596, "Ley que Regula el Régimen Jurídico de Canes". Se ha reportado que los índices persona:can y otras informaciones de interés como porcentaje de viviendas con canes, distribuciones de edades, sexos, etc., en cada ciudad latinoamericana difiere entre ellas (Málaga, 1991). Por ello, se indica que sería sumamente peligroso extrapolar resultados de una ciudad a otra. Y aún más que dentro de una ciudad, los índices difieren entre los distintos lugares que la forman, lo que de cierto grado refleja el desarrollo entre ciudades e intra ciudades (Málaga, 2010).

La Municipalidad de SMP, consciente de la necesidad de contar con esta información, ha respaldado un estudio realizado a través de IE. La razón por las que se eligió este medio es porque de esa forma se podía acceder a información de un gran número de hogares en un tiempo corto y con una menor inversión. Aunque el muestreo termina siendo por conveniencia y no se puede asegurar la aleatorización de la muestra debido a que la participación fue voluntaria, el volumen de encuestas recogidas $(n=1947)$ es la suficientemente grande por lo que siguiendo los criterios del Teorema del Limite Central, la muestra obtenida seguiría la distribución normal (Daniel, 2002) y por los tanto los resultados serían representativos de la población en estudio. 
La relación persona:can encontrada fue de 7:1, valor por encima de la relación utilizada por el sector salud para estimar la población de canes a vacunar contra la rabia. El número de canes estimados para la campaña VANCAN 2013 en SMP fue de 40413 para una población humana estimada de 556 021(OITE, 2013), lo que representa una relación can: persona 13,8:1. Ello indicaría que una población significativa de canes no estaría vacunada y consecuentemente se encontrarían en riesgo de adquirir la rabia, ante la eventual presencia de un can portador del virus y la aparición de un brote de rabia en la capital.

Según la OMS, para lograr un control y eventualmente la eliminación de la rabia, las campañas de vacunación deben ser anuales y alcanzar al menos un $70 \%$ de cobertura (WHO, 2013).Sin embargo, la "Norma Técnica de Salud Para la Prevención y Control de Rabia Humana en el Perú" (MINSA, 2008) establece que el número de canes a vacunar en campañas debe ser mínimo de un $80 \%$ de la población estimada y que las campañas deben realizarse en uno o dos días, con el propósito de generar una respuesta inmunológica capaz de interrumpir la transmisión de la rabia en la población de canes.

Por ello, los resultados encontrados en el estudio deberían de servir para revisar la planificación anual que se viene realizando actualmente para el VANCAN lo que conllevará a tener una mayor población de canes vacunada y protegida contra esta enfermedad, y consecuentemente disminuyendo el riesgo de transmisión de la rabia a la población humana.

La estimación real de la población de canes es de importancia en Salud Pública, ya que conforme aumenta la población de canes, más cercano será el contacto con el hombre incrementando el riesgo de transmisión de zoonosis (Güttler, 2005).Utilizando los datos del Censo del INEI para el 2007 y la proyección al 2013 realizada por la misma institución se observa que en solo seis años la tasa de crecimiento de la población de canes ha sido del $16 \%$. Esta tasa de crecimiento podría haberse mantenido también en la población de canes en el caso que la relación persona:can se hubiera estabilizado. Al respecto, Málaga (2010) a partir de la revisión de diversos estudios, menciona que se asume que la población canina crece a la misma velocidad que las humanas, lo que se ha confirmado en Lima Metropolitana en los estudios de 1970, 1974 y en 1985. En dichos estudios se ha obtenido la relación persona:can de 10:1, 10:1 y 11:1 respectivamente.
Por otro lado, Haro (2003) menciona que si el crecimiento de la población de canes se mantiene proporcional al de la población humana, se elevaría el riesgo de transmisión de enfermedades zoonóticas. Por ello se hace necesario desarrollar programas de control poblacional de canes, especialmente en aquellas poblaciones de menos recursos económicos, quienes resultan más vulnerables debido a que no disponen de presupuesto para esta actividad. Además, generalmente desconocen los riesgos que representan las zoonosis y tienen un acceso limitado a servicios de salud de calidad.

Estudios de estimación de poblaciones de canes en distritos de Lima son escasos. El año 1970 se realizó un estudio de las características de la población canina y felina de Lima Metropolitana. En él se encontró una relación de 9,37:1 para el distrito de SMP y un global para Lima de 10,26:1. Por aquellos años la población de canes para SMP y Lima fue estimada en 15099 y 300 543, respectivamente (Málaga, 1973). En años más recientes, un estudio a partir de encuestas en IE se realizó en el distrito de Comas (Lima, Perú), encontrando una relación persona:can de 5,7:1 (Soriano, 2014). Otro estudio a base de encuestas en viviendas realizado en la Comunidad de Jardines de Manchay, distrito de Pachacamac-Lima reportó la relación de 3,9:1 (Málaga et al., 2014)

En cuanto al porcentaje de hogares que poseen canes, se encontró la tenencia de estos animales en el $58,2 \%$ de los encuestados. Este resultado es similar a los encontrados en el distrito de Comas (Lima, Perú) donde el 60,5\% de viviendas reportó la tenencia de al menos un can (Soriano, 2014). Resultados similares también se han encontrado en ciudades de Chile, como Viña del Mar, con un 57\% (Morales et al., 2009), Chillán con 52,8\% (Véliz, 1993), Entre Lagos (Haro, 2003) y Chacao (Bravo, 2003), ambos situados en Valdivia, con 63\%; y en Santiago de Chile 63,2\% (Pavez, 2009). El promedio de canes por vivienda $(1,61)$ resultó ser también similar a otros estudios realizados en Chile. En Valdivia, por ejemplo, se encontró un promedio de 1,3 canes por vivienda (Bravo, 2003) y en Santiago de Chile 1.1 (Pavez, 2009). Esta información es de importancia en Salud Pública ya que a mayor cantidad de canes en hogares se presenta un mayor riesgo de contraer zoonosis (Figueroa, 2002).

Las diferencias encontradas en la proporción de viviendas con canes según el tipo de estas $(64,3 \%$ en casa y $35,6 \%$ en departamento) fueron similares a las encontradas en Santiago de Chile donde la tenencia de 
canes era de $69,7 \%$ en casas y $20,8 \%$ en departamento. Ello ha sido atribuido a que las personas que vivían en departamentos no se les permitía mantener canes y/o no contaban con una persona que pueda ocuparse del can, ya que la vivienda quedaba desocupada gran parte del día (Pavez, 2009).

La utilidad de canes como mascota fue lo que predominó en SMP obteniéndose resultados similares a los encontrados en Comas (Soriano, 2014) distrito vecino a SMP. En contraste, en Viña del Mar el porcentaje de utilidad de los canes como mascotas y la utilidad tanto de mascota como guardián $(42,4 \%$ y $41,4 \%$ respectivamente) fue más estrecha (Morales et al., 2009). Por otro lado, en Santiago de Chile la razón afectiva mostró un menor porcentaje $(22,4 \%)$ en relación a la utilidad de guardianía $(41,4 \%)$ ya que el problema de seguridad aquí toma mayor importancia que en Viña del Mar (Acuña, 1998).

El tiempo y tipo de confinamiento puede ser un indicador para evaluar la TRAC. El estudio encontró que el mayor porcentaje correspondió a la salida de canes bajo supervisión de sus dueños, seguidos de un confinamiento permanente y salida libre en algún momento del día. Estos resultados difieren de los obtenidos en Viña del Mar, donde un 77.5\% mantiene un confinamiento permanente, el $10.5 \%$ un confinamiento temporal y un $12 \%$ sin confinamiento (Morales et al., 2009). Otras ciudades como Valdivia (García, 1995) e Iquique (Luza, 1996) mostraron un menor control de confinamiento $(61,9 \%$ y $59.6 \%$ respectivamente) lo que indicaría una menor tenencia responsable de canes. En el distrito de Comas los valores de confinamiento fueron similares a los de SMP, $34.8 \%$ de canes sale bajo supervisión, el 27,1\% mantiene un confinamiento permanente y el $29,4 \%$ sale sin supervisión en algún momento del día (Soriano, 2014).

La distribución de la población de canes por sexo para SMP se asemeja a la de Viña del Mar (62\% machos - 38\% hembras) y Santiago de Chile $(49,1 \%$ machos - 32,8\% hembras). En general se prefiere un animal macho principalmente debido a las molestias que presentan las hembras en la época de celo (Gallardo, 1998; Cantillano, 2002).

La edad promedio de la población de canes en SMP (2.7 años) resultó menor a la población del Distrito de Comas que fue de 3,05 años (Soriano, 2014). En ambos casos se puede concluir que la población de canes es joven en contraste a las encontradas en promedio en algunas ciudades de Chile como Valdivia (5 años) y Viña del Mar (4.58 años) (Güttler, 2005; Morales et al, 2009); y en Argentina, como San Martin de los Andes (4,9 años) (Brusoni et al., 2007). Sin embargo, en Bogotá, Colombia el promedio se mostró cercano a los obtenidos en el Distrito de Comas y SMP, con un valor de 3,5 años (Codeisa, 1999).

La distribución de canes por raza no determinada fue mayor que los de raza definida. Estos porcentajes fueron similares a los encontrados en el distrito de Comas, en donde los canes mestizos se reportaron en un 55.3\% (Soriano, 2014). Este comportamiento también se encuentra en otros estudios. En ciudades de Chile, se observó canes mestizos en un $64,7 \%$ en Viña del Mar y $67,1 \%$ en Santiago de Chile. No se conoce la razón por este tipo de preferencias, pero puede estar asociado principalmente a su costo de mantenimiento (Güttler, 2005).

La preferencia de animales mestizos es probable que esté influenciada por las campañas de sensibilización promocionadas desde los gobiernos locales y ONGs, lo que estaría fomentando que personas que deseen tener una mascota decidan adoptar un can mestizo. Respecto al tamaño, en el estudio predominó el tamaño mediano y en menor proporción los de alzada grande. Esto se debería primordialmente al costo de su alimentación y al espacio que estos animales requieren (Güttler, 2005).

El uso de la esterilización como método de control reproductivo ha sido reportado en solo $11,6 \%$ de los tenedores de canes. Una proporción similar (9.9\%) también fue encontrada en el distrito de Comas (Soriano, 2014). Se debe tener presente que la población de canes está experimentando un crecimiento rápido principalmente por la dominancia de animales jóvenes, independientes y con bajo nivel de castración, que logran tener altas tasas de fertilidad y tasas bajas de mortalidad (Brusoni et al.,2007). Por eso, es importante fomentar campañas de esterilización, sobre todo en distritos de bajos recursos donde existe un menor conocimiento y prácticas de TRAC, para que con la ayuda de los gobiernos locales correspondientes y médicos veterinarios capacitados puedan acceder a esterilizar a los canes a bajo costo. Sin embargo, hay que tener en cuenta que estas campañas de esterilización deberían estar destinada solamente a familias de menores recursos previa evaluación de nivel socioeconómico, y así evitar una competencia desleal hacia los médicos veterinarios de práctica privada (Llalla, 2012). 
Entre los indicadores de TRAC se debe tener en cuenta que las prácticas sanitarias preventivas que los propietarios aplican a sus animales, entre ellas la desparasitación. Hay que considerar que el $20 \%$ de zoonosis corresponden a este tipo de enfermedades y su control se hace necesario (Rosas, 1997). El estudio reporta que la tasa de animales desparasitados de forma interna es menor que los desparasitados en forma externa. Esto se debería a que los dueños pueden tener mayor conocimiento y conciencia de una desparasitación externa, ya que puede visualizarse con mayor facilidad en el animal una infestación por pulgas, garrapatas o ácaros, este último debido a las lesiones en piel, y así el dueño puede tomar la decisión de controlarlos. En cambio, una parasitemia interna, no siempre es fácil de percatarse, sin embargo en muchos casos suele tener mayor importancia en la salud pública.

La tasa de mortalidad de las crías $(24,3 \%)$ alcanzó un nivel inferior al obtenido en el distrito de Comas (35,3\%) (Soriano, 2014), pero muy similar al encontrado en Viña del Mar (23\%). En este caso se menciona que el aplastamiento de crías por la madre sería la principal causa de mortalidad (Morales et al., 2009).

Los resultados del estudio pueden ser considerados como una línea de base a partir de la cual las autoridades de salud y gobierno local pueden mejorar la planificación, tanto del programa de vacunación antirrábica como el que involucra la aplicación de la Ley No 27596, "Ley que Regula el Régimen Jurídico de Canes", y consecuentemente mejorar su cobertura e impacto.

Se concluye que la estimación de la población de canes con dueño en el distrito de SMP muestra que la población de canes vacunados se encontraría por debajo de los niveles aceptados para mantener a esta población protegida contra la rabia, por lo que se sugiere que se replante los actuales criterios para estimar la población a vacunar contra dicha enfermedad en las campañas VANCAN.

\section{Correspondencia:}

Debhora Arauco Serquen cristi_2105@hotmail.com

\section{REFERENCIAS}

1. Acha P, Szyfres B. 2001. Zoonosis y enfermedades transmisibles comunes al hombre y los animales. Washington: OPS/OMS. Publicación científica $\mathrm{N}^{\circ}$ 580. 3ra ed. 398 p.

2. Acuña P. 1998. Demografía canina y felina en el Gran Santiago 1997. Tesis de Médico Veterinario. Chile: Universidad de Chile.81 p.

3. Álvarez E, Domínguez J. 2001. Programa para el control integral de la población canina. AMMVEPE 2001; 12: 83-91.

4. Bravo Y. 2003. Censo de la población canina y estimación del grado de consulta hospitalaria por mordedura en la localidad de Chacao, provincia de Chiloe. Tesis de Médico Veterinario. Chile, Valdivia: Universidad Austral de Chile. 37 p.

5. Brusoni C., Dezzotti A., Fernández J., Lara J. 2007. Tamaño y estructura de la población canina en San Martín de los Andes (Neuquén). Analecta Veterinaria 27 (1): 11-23.

6. Butcher R. 2000. La implementación de programas de control de animales vagos, los efectos de las diferencias económicas y culturales. MEVEPA 14, 40-46.

7. Cantillano J. 2002. Características demográficas de la población canina en Quemchi, Provincia de Chiloé, nivel de conocimiento de los propietarios sobre enfermedades zoonóticas y el manejo de sus caninos. Tesis de Médico Veterinario. Chile, Valdivia: Universidad Austral de Chile. 35 p.

8. [CODEISA] Secretaria distrital de Salud,. 1999. Comportamiento de la población canina del Distrito Capital analizando tasa de fecundidad, natalidad y mortalidad y la relación hombre-animal (Informe final). Bogotá. 252p.9. Daniel W. 2002. "Bioestadística. Bases para el análisis de las Ciencias de la Salud". Ed. Limusa.México.

10. Figueroa J. 2002. Censo de la población canina y felina y estimación del grado de consulta hospitalaria por mordeduras en la ciudad de Lago Ranco. Tesis de Médico Veterinario. Chile, Valdivia: Universidad Austral de Chile. 44 p.

11. Gallardo C. 1998. Estudio demográfico de la población canina en la localidad de Canela Baja, Provincia de Choapa, IV Región. Tesis de Médico Veterinario. Chile, Valdivia: Universidad Austral de Chile. 48 p.

12. García H. 1995. Estimación demográfica de la población canina en la ciudad de Valdivia. Tesis de Médico Veterinario. Chile, Valdivia: Universidad Austral de Chile. 73 p.

13. Güttler V. 2005. Análisis de algunas características de la población canina relacionadas con mordeduras e hidatidosis humana en la provincia de Valdivia. 
Tesis de Médico Veterinario. Chile, Valdivia: Universidad Austral de Chile. 60p

14. Ley $\mathrm{N}^{\circ} 27596$. Ley que regula el régimen jurídico de canes. Diario Oficial El Peruano Lima, 14 de diciembre de 2001.

15. Llalla H. 2012. Caracterización de la tenencia de mascotas y evaluación de conocimientos, actitudes y prácticas asociadas a riesgos de zoonosis y accidentes entre escolares de educación secundaria en tres distritos de Lima Metropolitana. Tesis de Maestría. Lima, Perú: Universidad Peruana Cayetano Heredia. 75 p.

16. Luza R. 1996. Características demográficas de la población canina en la ciudad de Iquique. Tesis de Médico Veterinario. Chile, Valdivia: Universidad Austral de Chile. 62 p.

17. Málaga H. 1973. Características de la población canina y felina de Lima Metropolitana. Ministerio de Salud. Lima-Perú. 32p.

18. Málaga H. 1991. Perspectivas de investigación en zoonosis. Revista científica, FCV de Luz. 1(1). Disponible en: http://www.saber.ula.ve/ bitstream/123456789/23650/2/articulo 7.pdf

19. Málaga H. 2010.Epidemiología Veterinaria. 2da ed. Universidad Peruana Cayetano Heredia. 184p.

20. Málaga H, Piña J, Denegri A, Stefan T, Cedamanos, D, Alvarado D. Taipe C. 2014. Priorización de problemas de salud comunitaria en una localidad de Lima Metropolitana en el marco del programa de Municipios Saludables. Revista Peruana de Epidemiología, 18(2), 1-4.

21.Martin W, Meek A, Willeberg P. 1997. Epidemiología Veterinaria. Principios y métodos. Editorial Acribia. Zaragoza, España. 398 p.

22. [MINSA] Ministerio de Salud. 2008. Norma Técnica de Salud para la Prevención y Control de la Rabia Humana en el Perú. Lima: MINSA. 102p.

23. Montes L. 1966. Estudio de la población canina de Santiago, su densidad y composición. Tesis Facultad de Ciencias Pecuarias y Medicina Veterinaria. Universidad de Chile. 73p.

24. Morales M, Varas C, Ibarra L.2009. Caracterización demográfica de la población de perros de Viña del Mar, Chile. Archivos de medicina veterinaria 41(1): 89-95.

24. [OIE] Organización Mundial de Sanidad Animal. 2009. Directrices sobre el control de las poblaciones de perros vagabundos. París: OIE. Volumen 1: Consideraciones generales. 19p.

26. [OITE] Unidad de Estadística de la Oficina de Informática, Telecomunicaciones y Estadística. 2013. Campaña VANCAN: Canes Vacunados Contra La Rabia Según
Establecimientos, Microredes y Redes de Salud. Lima: OITE. Dirección de Salud V Lima Ciudad.

27. Ortega A. 2001. La sobrepoblación canina: un problema con repercusiones potenciales para la salud humana. Rev Biomed 12: 290-291.

28. Pavez C.2009. EstimaciónyAlgunas Características de la Población Canina con Dueño en 34 comunas de la Ciudad de Santiago. Tesis de Médico Veterinario. Chile, Santiago de Chile: Universidad Iberoamericana de Ciencias y Tecnología. $30 \mathrm{p}$.

29. [REDIPRA 13] $13^{\circ}$ Reunión de Directores de los Programas Nacionales de Control de Rabia en América Latina. 2010. Conclusiones de la REDIPRA 13 para evitar la Rabia en las Américas. Buenos Aires: REDIPRA. 29p.

30. [REDIPRA 14] $14^{\circ}$ Reunión de Directores de los Programas Nacionales de Control de Rabia en América Latina. 2013. Plan de Acción para la Eliminación de la Rabia Humana transmitida por Perros. Lima: REDIPRA. Salud Pública Veterinaria. $11 \mathrm{p}$.

31. Rosas C. 1997. Revisión bibliográfica de las principales zoonosis parasitarias en Chile; período 1977- 1994. Tesis de Médico Veterinario. Chile, Valdivia: Universidad Austral de Chile. 83 p.

32. Soriano JF. 2014. Estimación de la población de canes con dueño en el distrito de Comas, LimaPerú. Tesis de Médico Veterinario Zootecnista. Perú, Lima: Universidad Peruana Cayetano Heredia. 39 p.

33. [WSPA] World Society for the Protection of Animals. 2007. Guía Para el Manejo Humanitario de Poblaciones Caninas. Londres: WSPA. 24p.

34. [WHO] World Health Organization. 2013. WHO Expert Committee on rabies. Geneva: WHO. Technical Report Series. 150p.

35. Veliz C. 1993. Estudio de algunas características de la población canina del área urbana de la ciudad de Chillán. Tesis de Médico Veterinario. Chile, Concepción: Universidad de Concepción. $104 \mathrm{p}$.

Recibido 27/07/2014

Aceptado 04/02/2015 